\title{
EFICÁCIA DOS HERBICIDAS BENTAZON 400/ACIFLUORFEN 170 E BENTAZON 400/ACIFLUORFEN 140 NO CONTROLE DE PLANTAS DANINHAS LATIFOLIADAS, EM PÓS-EMERGÊNCIA, NA CULTURA DA SOJA
}

\author{
Júlio P. Laca-Buendia ${ }^{1}$ Joel C. Ferreira ${ }^{2}$ \\ Engenheiro Agrônomo, M. Sc., Pesquisador. EPAMIG. Av. Amazonas, 115 - Sala 619. Belo Horizonte, MG 30180-902 \\ 2 Engenheiro Agrônomo, Pesquisador. EPAMIG/FEGV. Caixa Postal 351. Uberaba, MG 38001-970
}

\begin{abstract}
RESUMO
O objetivo do ensaio foi avaliar a eficácia dos herbicidas bentazon 400/acifluorfen 170 e bentazon 400/acifluorfen 140, isolados e em mistura com óleo mineral (Assist), em pós-emergência inicial, para o controle de plantas daninhas latifoliadas na cultura da soja, cv. 'Garimpo', em Latossolo Vermelho-Escuro, textura média, em área de Cerrado. Os tratamentos foram: bentazon 400/acifluorfen $170(570,684 \mathrm{e} 855 \mathrm{~g} / \mathrm{ha})$, bentazon 400/acifluorfen 170+óleo mineral (684+378 g/ha), bentazon 400/ acifluorfen $140(540,648 \mathrm{e} 810 \mathrm{~g} / \mathrm{ha})$, bentazon 400/acifluorfen 140+óleo mineral (648+378 g/ha), fomesafen/fluazifop-butyl (500 $\mathrm{g} / \mathrm{ha}$ ) e testemunha capinada e sem capina. Na produtividade de grãos nenhum dos herbicidas estudados apresentou diferenças com relação à testemunha capinada. Porém a testemunha sem capina apresentou diferenças em relação a todos os demais tratamentos, com a produção de $813 \mathrm{~kg} / \mathrm{ha}$. Para a densidade de plantas por ocasião da colheita, altura média das plantas, altura média de inserção da primeira vagem e peso de 100 sementes, não foram detectadas diferenças entre os tratamentos.Verificouse que Amaranthus viridis (caruru) foi controlado por todos os herbicidas, com índices de controle acima de $95 \%$, até os 21 dias após a aplicação. Para Nicandra physaloides (joá-de-capote), todos os herbicidas apresentaram um excelente controle, com índice de $100 \%$ desde os 7 até os 21 dias após a aplicação. Para Sida rhombifolia (vassoura), todos os herbicidas apresentaram índices de controle superiores a $97 \%$, até 21 dias depois da aplicação. Na primeira avaliação verificou-se leve toxicidade às plantas causada pelas misturas fomesafen/fluazifop-butyl, bentazon 400/acifluorfen 140+óleo mineral (684+378 g/ha) e bentazon 400/acifluorfen 140. Na segunda avaliação, 15 dias após a aplicação, não era visível qualquer sintoma de toxicidade.
\end{abstract}

Palavras-chave: mistura formulada de herbicidas, Glycine max, Amaranthus viridis, Nicandra physaloides, Sida rhombifolia.

\section{ABSTRACT \\ Postemergence broadleaf weed control in soybean with bentazon 400/acifluorfen 170 and bentazon 400/acifluorfen 140}

The objective of the experiment was to evaluate the efficacy of herbicides bentazon 400/acifluorfen 170 and bentazon 400/acifluorfen 140, alone and in mixture with mineral oil (Assist) in early postemergence control of weeds in soybean. Treatments were: bentazon 400/acifluorfen $140(570,684$ and $855 \mathrm{~g} / \mathrm{ha})$, bentazon 400/acifluorfen 170+mineral oil ( $684+378 \mathrm{~g} / \mathrm{ha})$, bentazon 400/acifluorfen $140(540,648$ and $810 \mathrm{~g} / \mathrm{ha})$, bentazon 400/acifluorfen 140+mineral oil (648+378 g/ha) and fomesafen/fluazifopbutyl $(500 \mathrm{~g} / \mathrm{ha})$ and a weedy and a weeded control. The results showed no difference in grain productivity between the herbicide treatments and the weeded control. However the weedy control showed differences in relation to all treatments, with productivity of $813 \mathrm{~kg} / \mathrm{ha}$. Soybean density at harvesting, plant height, first pod insertion height and weight of 100 seeds, showed no difference between treatments. Amaranthus viridis was controled by all herbicides, with $95 \%$ control or more until 
Júlio P. Laca-Buendia

21 days after aplication. Nicandra physaloides was also controlled by all herbicides from 7th to 21st day after spraying. In Sida rhombifolia, all herbicides showed index control higher than $97 \%$ until 21 days after aplication. Seven days after herbicide spraying it was observed negligible toxicity to soybean plants. Seven days later there was no toxicity.

Key words: herbicide misture, Glycine max, Amaranthus viridis, Nicandra physaloides, Sida rhombifolia.

\section{INTRODUÇÃO}

A descoberta da região do Cerrado como uma das mais promissoras para a expansão e cultivo da soja no Brasil levaram os órgãos de pesquisa agropecuária a intensificar seus trabalhos visando a determinação de melhores técnicas de cultivo do produto, como também de sua melhor aplicação dentro do sistema agrícola da região.

O controle químico de plantas daninhas, está fundamentado principalmente no uso de herbicidas de aplicação ao solo. Com o surgimento de herbicidas de aplicação em pósemergência e seletivos para a cultura da soja, diversos herbicidas latifolicidas de pós-emergência vem sendo desenvolvidos, com eficiente controle das plantas daninhas.

A concorrência das plantas daninhas com a cultura pode reduzir a produção em até $90 \%$ (Blanco et al., 1973). Para as condições de Cerrado, no Triângulo Mineiro, o melhor rendimento foi obtido quando se manteve a cultura livre da competição durante os primeiros 40 dias após a emergência (Maia et al., 1992b).

Indicações de herbicidas na cultura da soja para o controle das plantas daninhas latifoliadas, aplicados na pós-emergência, tem sido feitas por vários pesquisadores (Maia et al., 1992a; Machado Neto et al., 1984; Melhorança, 1984; Braga, 1988; Barros, 1993; Stroher \& Haden, 1993 e Laca-Buendia \& Rezende, 1993).

O objetivo do ensaio de campo foi avaliar a eficácia dos herbicidas bentazon 400/acifluorfen 170 e bentazon 400/ acifluorfen 140, isolados e em mistura com óleo mineral, em pós-emergência inicial, para o controle de plantas daninhas latifoliadas na cultura da soja, em Cerrado.

\section{MATERIAL E MÉTODOS}

O ensaio de campo foi instalado na Fazenda da Escola Agrotécnica Federal de Uberaba, MG, no ano agrícola de 1994/ 95, em solo de Cerrado do tipo Latossolo Vermelho-Escuro, textura média, com $67 \%$ de areia, $24 \%$ de argila, $9 \%$ de limo, $1,6 \%$ de matéria orgânica , $\mathrm{pH}$ (água) =6,3, 17 ppm de P, 90 ppm de K, 2 meq/100cc de Ca, 1 meq/100ml de $\mathrm{Mg}$ e $68 \%$ de saturação de bases da CTC a pH=7.

A cultivar usada foi a 'Garimpo', semeada em 28/12/94, utilizando-se o espaçamento de $0,5 \mathrm{~m}$ entre fileiras, semeadura de tração mecânica e densidade de 25 sementes por metro linear.
A adubação de plantio usada foi de $400 \mathrm{~kg} /$ ha de NPK, utilizando-se a fórmula 0:20:20.

O ensaio foi instalado no desenho experimental de blocos casualizados, com quatro repetições em parcelas de $2,0 \mathrm{~m}$ $\mathrm{x} 7,0 \mathrm{~m}=14,0 \mathrm{~m}^{2}$, sendo colhidas as duas fileiras centrais, eliminando-se $0,5 \mathrm{~m}$ nas extremidades, constituindo uma área útil de $6,0 \mathrm{~m}^{2}$.

Os herbicidas (Tabela 1), foram aplicados em pós-emergência, em 14/01/95, quando a soja apresentava-se com 3 a 4 trifólios e as plantas daninhas latifoliadas com 2 a 4 folhas. Foi utilizado um pulverizador costal pressurizado $\mathrm{aCO}_{2}$, dotado de barra, com quatro bicos do tipo leque $110.04 \mathrm{VS} \mathrm{a} 0,5 \mathrm{~m}$ do alvo, com volume de calda de 287 1/ha, usando-se uma pressão constante de $4 \mathrm{~kg} / \mathrm{cm}^{2}$, entre $6: 35$ h e 7:00 horas. O tempo se apresentava ensolarado, sem vento e o solo úmido (112 mm de chuva durante os dez dias antes da aplicação), com temperatura do ar de $32^{\circ} \mathrm{C}$ e umidade relativa do ar de $66 \%$. As precipitações pluviais ocorridas no transcorrer do ensaio encontram-se discriminadas na Tabela 2.

Foram realizadas três capinas manuais nas parcelas testemunhas capinadas, para o controle das plantas daninhas, em 17/01/95, 29/01/95 e 19/02/95.

Para o controle do percevejo-verde (Nezara viridula) foram realizadas duas aplicações do inseticida Hamidop na dose de $500 \mathrm{ml} / \mathrm{ha}$, sendo a primeira em 05/03/95 e a segunda em 13/04/95.

As avaliações visuais de toxicidade às plantas de soja foram realizadas aos 7 e 15 dias após a aplicação dos herbicidas, através da escala visual EWRC ( 1 a 9), em que 1 corresponde a nenhuma injúria e 9 à morte da planta.

Aos 7, 14, e 21 dias após a aplicação realizaram-se avaliações da eficácia de controle, utilizando-se a escala percentual de $0 \%$ a $100 \%$, onde $0 \%$ corresponde a nenhum controle e $100 \%$ ao controle total (excelente) para cada espécie de planta daninha encontrada nas parcelas dos tratamentos com herbicidas e nas testemunhas. Na testemunha sem capina foi realizada a contagem por espécie dentro da área útil de cada parcela em 1,0 m².

As espécies encontradas na área em estudo foram: Amaranthus viridis (caruru), com 29 indivíduos $/ \mathrm{m}^{2}$, Nicandra physaloides (joá-de-capote), com 11 indivíduos $/ \mathrm{m}^{2}$ e Sida rhombifolia (vassoura), com 19 indivíduos $/ \mathrm{m}^{2}$.

Quando as plantas atingiram a maturação de colheita, em 08/05/95, realizou-se a pesagem dos grãos da área útil e contouse o número de plantas em $3,0 \mathrm{~m}^{2}$ de cada parcela. A altura média das plantas e a altura de inserção da primeira vagem foram tomadas de 10 plantas ao acaso por parcela. Foram escolhidas ao acaso 100 sementes para determinar seu peso. 
Tabela 1. Tratamentos utilizados no experimento. Uberaba - MG, 1994/95.

\begin{tabular}{|c|c|c|c|c|}
\hline \multirow[b]{2}{*}{ Produto técnico } & \multicolumn{2}{|c|}{ Doses } & \multirow{2}{*}{$\begin{array}{l}\text { Produto } \\
\text { Comercial }\end{array}$} & \multirow{2}{*}{$\begin{array}{c}\text { Concentração } \\
(\mathrm{g} / \mathrm{l})\end{array}$} \\
\hline & $\begin{array}{c}\text { Produto Técnico } \\
\text { (g/ha) }\end{array}$ & $\begin{array}{c}\text { Produto Comercial } \\
\text { (l/ha) }\end{array}$ & & \\
\hline Bentazon 400/acifluorfen 170 & 570 & 1,0 & Volt & 570 \\
\hline Bentazon 400/acifluorfen 170 & 684 & 1,2 & Volt & 570 \\
\hline Bentazon 400/acifluorfen 170 & 855 & 1,5 & Volt & 570 \\
\hline Bentazon 400/acifluorfen $170+$ o. m. & $684+378$ & $1,2+0,5$ & Volt + Assist & $570+189$ \\
\hline Bentazon 400/acifluorfen 140 & 540 & 1,0 & Gunner & 540 \\
\hline Bentazon 400/acifluorfen 140 & 648 & 1,2 & Gunner & 540 \\
\hline Bentazon 400/acifluorfen 140 & 810 & 1,5 & Gunner & 540 \\
\hline Bentazon 400/acifluorfen $140+$ o. m. & $648+378$ & $1,2+0,5$ & Gunner+Assist & $540+189$ \\
\hline Fomesafen/fluazifop-butyl & 500 & 2,0 & Fusiflex & 250 \\
\hline Testemunha capinada & -- & -- & --- & --- \\
\hline Testemunha sem capina & -- & -- & -- & -- \\
\hline
\end{tabular}

Tabela 2. Precipitação pluvial, em mm, durante o decorrer do ensaio. Uberaba - MG, 1994/95.

\begin{tabular}{lcccc}
\hline Mês & $\mathbf{1}^{\mathbf{0}}$ Decêndio & $\mathbf{2}^{\mathbf{0}}$ Decêndio & $\mathbf{3}^{\mathbf{0}}$ Decêndio & Total \\
\hline Dezembro & 0,0 & 0,0 & 107,8 & 107,8 \\
Janeiro & 112,0 & 26,2 & 102,6 & 240,8 \\
Fevereiro & 185,2 & 81,8 & 31,4 & 298,4 \\
Março & 48,2 & 43,8 & 78,2 & 170,2 \\
Abril & 32,4 & 33,8 & 31,0 & 127,2 \\
Maio & 85,8 & 0,0 & 0,0 & 85,8 \\
\hline Total & & & & $\mathbf{0 3 0 , 2}$ \\
\hline
\end{tabular}

\section{RESULTADOS E DISCUSSÃO}

As condições locais onde foi conduzido o ensaio de campo foram ótimas para o desenvolvimento da cultura, bem como para a ocorrência das plantas daninhas.

Com relação á densidade de plantas por ocasião da colheita, altura média das plantas, altura média de inserção da primeira vagem e peso de 100 sementes, não foram detectadas diferenças entre os tratamentos testados (Tabelas 3 e 4).

Houve diferenças significativas na produção de grãos, sendo que nenhum dos herbicidas testados apresentou diferenças com relação á testemunha capinada e os valores oscilaram entre 1653 e 1246 kg/ha. Porém a testemunha sem capina apresentou diferenças em relação a todos os demais tratamentos, com a produção de 813 $\mathrm{kg} / \mathrm{ha}$.
Na primeira avaliação foi observada toxicidade às plantas, causada pelo herbicida fluazifop-butil/fomesafen, sendo que a nota apresentada foi 4,8 (15\%=aceitável na prática), seguida pela aplicação de bentazon 400/acifluorfen $140+$ óleo mineral $(684+378 \mathrm{~g} / \mathrm{ha})$, com $4,5(10 \%$ a $15 \%=$ moderada a aceitável na prática). Os outros herbicidas apresentaram valores iguais a $2,0(2 \%=$ traços $)$, sendo que na segunda avaliação, após 15 dias da aplicação, todos os herbicidas estudados apresentaram nota $1,0(0 \%=$ nenhuma injúria) (Tabela 4$)$.

O caruru foi controlado por todos os herbicidas, os quais apresentaram índices de controle iguais ou acima de $95 \%$ aos 21 dias após a aplicação (DAA) (Tabela 5). Para o joáde-capote, todos os herbicidas estudados apresentaram um excelente controle, com índices de $100 \%$ desde os 7 até os 21 dias após a aplicação (Tabela 5). Para a vassoura, todos os herbicidas apresentaram índices de controle igual ou acima de 97\%, aos 21 dias após a aplicação (Tabela 6). 
Tabela 3. Efeito de herbicidas aplicados em pós-emergência na cultura da soja. Uberaba-MG, 1994/95.

\begin{tabular}{|c|c|c|c|c|}
\hline Tratamento & $\begin{array}{l}\text { Dose } \\
\text { (g/ha) }\end{array}$ & $\begin{array}{l}\text { Densidade final } \\
\text { (plantas/3 m²) }\end{array}$ & $\begin{array}{c}\text { Produção } \\
\text { (kg /ha) }\end{array}$ & $\begin{array}{l}\text { Altura da } \\
\text { planta } \\
(\mathrm{cm})\end{array}$ \\
\hline Bentazon 400/acifluorfen 170 & 570 & $123 a^{(1)}$ & $1281 \mathrm{a}$ & $88,4 a$ \\
\hline Bentazon 400/acifluorfen 170 & 684 & $112 \mathrm{a}$ & $1296 a$ & $91,8 \mathrm{a}$ \\
\hline Bentazon 400/acifluorfen 170 & 855 & $111 \mathrm{a}$ & $1458 \mathrm{a}$ & $83,8 \mathrm{a}$ \\
\hline Bentazon 400/acifluorfen $170+$ o. m. & $684+378$ & $126 a$ & $1446 a$ & $81,8 \mathrm{a}$ \\
\hline Bentazon 400/acifluorfen 140 & 540 & $141 \mathrm{a}$ & $1340 \mathrm{a}$ & $85,3 a$ \\
\hline Bentazon 400/acifluorfen 140 & 684 & $144 a$ & $1246 a$ & $84,6 a$ \\
\hline Bentazon 400/acifluorfen 140 & 810 & $129 \mathrm{a}$ & $1518 \mathrm{a}$ & $87,4 \mathrm{a}$ \\
\hline Bentazon 400/acifluorfen $140+$ o. m. & $684+378$ & $147 \mathrm{a}$ & $1266 a$ & $89,9 a$ \\
\hline Fomesafen/fluazifop-butyl & 500 & $131 \mathrm{a}$ & $1421 \mathrm{a}$ & $84,6 a$ \\
\hline Testemunha capinada & --- & $151 \mathrm{a}$ & $1653 a$ & $87,3 \mathrm{a}$ \\
\hline Testemunha sem capina & --- & $99 a$ & $813 b$ & $77,3 \mathrm{a}$ \\
\hline Média & & 129 & 1340 & 85,6 \\
\hline Valor de F & & $2,11 \mathrm{~ns}$ & $8,74^{* *}$ & $0,94 \mathrm{~ns}$ \\
\hline DMS & & -- & 429 & -- \\
\hline C.V.(\%) & & 17,7 & 13,2 & 9,6 \\
\hline
\end{tabular}

Tabela 4. Efeito de herbicidas aplicados em pós-emergência na cultura da soja. Uberaba-MG, 1994/95.

\begin{tabular}{|c|c|c|c|c|c|}
\hline \multirow{2}{*}{ Tratamento } & \multirow{2}{*}{$\begin{array}{l}\text { Dose } \\
\text { (g/ha) }\end{array}$} & \multirow{2}{*}{$\begin{array}{c}\text { Altura } \\
\text { inserção } 1^{\mathrm{a}} \\
\text { vagem } \\
(\mathrm{cm})\end{array}$} & \multirow{2}{*}{$\begin{array}{l}\text { Peso de } 100 \\
\text { sementes } \\
\text { (g) }\end{array}$} & \multicolumn{2}{|c|}{$\begin{array}{l}\text { Fitotoxicidade } \\
\text { (EWRC } 1 \text { a 9) }\end{array}$} \\
\hline & & & & 7 DAA $\left({ }^{*}\right)$ & 14 DAA \\
\hline Bentazon 400/acifluorfen 170 & 570 & 12,6 & 14,9 & $1,8 \mathrm{bc}$ & 1,0 \\
\hline Bentazon 400/acifluorfen 170 & 684 & 12,1 & 15,5 & $1,2 b c$ & 1,0 \\
\hline Bentazon 400/acifluorfen 170 & 855 & 12,4 & 15,1 & $1,2 \mathrm{bc}$ & 1,0 \\
\hline Bentazon 400/acifluorfen $170+$ o. m. & $684+378$ & 12,7 & 14,8 & $2,8 \mathrm{~b}$ & 1,0 \\
\hline Bentazon 400/acifluorfen 140 & 540 & 12,7 & 15,8 & $1,2 b c$ & 1,0 \\
\hline Bentazon 400/acifluorfen 140 & 684 & 12,4 & 13,4 & $1,2 b c$ & 1,0 \\
\hline Bentazon 400/acifluorfen 140 & 810 & 12,3 & 14,4 & $1,2 \mathrm{bc}$ & 1,0 \\
\hline Bentazon 400/acifluorfen $140+$ o. m. & $684+378$ & 12,9 & 14,5 & $1,2 \mathrm{bc}$ & 1,0 \\
\hline Fomesafen/fluazifop-butyl & 500 & 11,8 & 15,6 & $4,8 \mathrm{a}$ & 1,0 \\
\hline Testemunha capinada & --- & 11,7 & 14,8 & $1,0 \mathrm{c}$ & 1,0 \\
\hline Testemunha sem capina & -- & 13,1 & 15,3 & $1,0 \mathrm{c}$ & 1,0 \\
\hline Média Geral & & 12,4 & 14,9 & 2,0 & 1,0 \\
\hline Valor de F & & $0,67 \mathrm{~ns}$ & $1,95 \mathrm{~ns}$ & $18,50 * *$ & \\
\hline DMS & & -- & -- & 1,6 & \\
\hline C.V. $(\%)$ & & 8,4 & 6,4 & 32,2 & \\
\hline
\end{tabular}


Tabela 5. Percentagem de controle de Amaranthus viridis, e Nicandra physaloides através da aplicação de herbicidas em pós-emergência, na cultura da soja. Uberaba-MG, 1994/95.

\begin{tabular}{|c|c|c|c|c|c|c|c|}
\hline \multirow{2}{*}{ Tratamentos } & \multirow{2}{*}{$\begin{array}{l}\text { Doses } \\
\text { (g/ha) }\end{array}$} & \multicolumn{3}{|c|}{ Amaranthus viridis ${ }^{(1)}$} & \multicolumn{3}{|c|}{ Nicandra physaloides ${ }^{(2)}$} \\
\hline & & 7 DAA & 14 DAA & 21 DAA & 7 DAA & 14 DAA & $21 \mathrm{DAA}$ \\
\hline Bentazon 400/acifluorfen 170 & 570 & $75 b^{(3)}$ & $75 b$ & $95 b$ & 100 & 100 & 100 \\
\hline Bentazon 400/acifluorfen 170 & 684 & $87 \mathrm{ab}$ & $82 \mathrm{ab}$ & $98 \mathrm{ab}$ & 100 & 100 & 100 \\
\hline Bentazon 400/acifluorfen 170 & 855 & $87 \mathrm{ab}$ & $85 \mathrm{ab}$ & $97 \mathrm{ab}$ & 100 & 100 & 100 \\
\hline Bentazon 400/acifluorfen $170+$ o. m. & $684+378$ & $90 \mathrm{ab}$ & $93 \mathrm{ab}$ & $97 b$ & 100 & 100 & 100 \\
\hline Bentazon 400/acifluorfen 140 & 540 & $85 \mathrm{ab}$ & $85 \mathrm{ab}$ & $96 b$ & 100 & 100 & 100 \\
\hline Bentazon 400/acifluorfen 140 & 684 & $85 \mathrm{ab}$ & $87 \mathrm{ab}$ & $98 \mathrm{ab}$ & 100 & 100 & 100 \\
\hline Bentazon 400/acifluorfen 140 & 810 & $87 \mathrm{ab}$ & $90 \mathrm{ab}$ & $96 b$ & 100 & 100 & 100 \\
\hline Bentazon 400/acifluorfen $140+o . \mathrm{m}$. & $684+378$ & $89 \mathrm{ab}$ & $92 \mathrm{ab}$ & $97 b$ & 100 & 100 & 100 \\
\hline Fomesafen/fluazifop-butyl & 500 & $85 \mathrm{ab}$ & $92 \mathrm{ab}$ & $95 b$ & 100 & 100 & 100 \\
\hline Testemunha capinada & --- & $100 \mathrm{a}$ & $100 \mathrm{a}$ & $100 \mathrm{a}$ & 100 & 100 & 100 \\
\hline Testemunha sem capina & --- & $0 \mathrm{c}$ & $0 \mathrm{c}$ & $0 \mathrm{~b}$ & 0 & 0 & 0 \\
\hline $\mathrm{N}^{\circ}$ de indivíduos $/ \mathrm{m}^{2}$ & & 29,9 & 27,5 & 24,8 & 12 & 22 & 21 \\
\hline Valor de F & & $16,48^{* *}$ & $24,13 * *$ & $230,8 * *$ & --- & --- & --- \\
\hline DMS & & 27,2 & 23,6 & 7,9 & --- & --- & --- \\
\hline C.V. $(\%)$ & & 17,8 & 14,3 & 4,4 & --- & --- & --- \\
\hline
\end{tabular}

DAA = dias após a aplicação.

(1) Dados transformados em Arco Seno $\sqrt{x / 100}$ para efetuar a análise da variância

(2) Não foi necessário realizar análise estatísticas para determinar diferenças entre médias dos tratamentos estudados.

${ }^{(3)}$ As médias na mesma coluna seguidas pela mesma letra, não diferem estatisticamente entre si, pelo teste de Tukey ao nível de $5 \%$ de probabilidade.

o. m. =óleo mineral

Tabela 6. Percentagem de controle de Sida rhombifolia, através da aplicação de herbicidas em pós-emergência, na cultura da soja. Uberaba-MG, 1994/95.

\begin{tabular}{|c|c|c|c|c|}
\hline \multirow{2}{*}{ Tratamento } & \multirow{2}{*}{$\begin{array}{l}\text { Dose } \\
\text { (g/ha) }\end{array}$} & \multicolumn{3}{|c|}{ Sida rhombifolia ${ }^{(2)}$} \\
\hline & & $7 \mathbf{D A A}^{1}$ & 14 DAA & $21 \mathrm{DAA}$ \\
\hline Bentazon 400/acifluorfen 170 & 570 & $87 \mathrm{~cd}^{(3)}$ & $91 \mathrm{~b}$ & $97 \mathrm{a}$ \\
\hline Bentazon 400/acifluorfen 170 & 684 & $96 a b c$ & $97 \mathrm{ab}$ & $98 \mathrm{a}$ \\
\hline Bentazon 400/acifluorfen 170 & 855 & $97 \mathrm{ab}$ & $98 \mathrm{ab}$ & $99 a$ \\
\hline Bentazon 400/acifluorfen $170+$ o. m. & $684+378$ & $93 \mathrm{abc}$ & $95 \mathrm{ab}$ & $100 \mathrm{a}$ \\
\hline Bentazon 400/acifluorfen 140 & 540 & $90 \mathrm{bcd}$ & $96 a b$ & $98 \mathrm{a}$ \\
\hline Bentazon 400/acifluorfen 140 & 684 & $100 \mathrm{a}$ & $99 \mathrm{ab}$ & $99 \mathrm{a}$ \\
\hline Bentazon 400/acifluorfen 140 & 810 & $96 a b c$ & $98 \mathrm{ab}$ & $99 \mathrm{a}$ \\
\hline Bentazon 400/acifluorfen $140+$ o. m. & $684+378$ & $92 \mathrm{bcd}$ & $96 \mathrm{ab}$ & $99 a$ \\
\hline Fomesafen/fluazifop-butyl & 500 & $82 \mathrm{~d}$ & $92 b$ & $97 \mathrm{a}$ \\
\hline Testemunha capinada & --- & $100 \mathrm{a}$ & $100 \mathrm{a}$ & $100 \mathrm{a}$ \\
\hline Testemunha sem capina & --- & $0 \mathrm{e}$ & $0 \mathrm{c}$ & $0 \mathrm{~b}$ \\
\hline $\mathrm{N}^{0}$ de individuos $/ \mathrm{m}^{2}$ & & 19 & 16 & 15 \\
\hline Média Geral & & 71,4 & 73,5 & 77,6 \\
\hline Valor de F & & $76,8 * *$ & $96,2 * *$ & $145,9 * *$ \\
\hline C.V. $(\%)$ & & 8,0 & 6,9 & 5,5 \\
\hline $\begin{array}{l}\text { (1) } \mathrm{DAA}=\text { dias após a aplicação } \\
{ }^{(2)} \text { Dados transformados em Arco seno } \sqrt{ } \\
{ }^{(3)} \text { As médias na mesma coluna seguidas péla } \mathrm{m} \\
\text { o. m. = óleo mineral }\end{array}$ & nestatis & . & 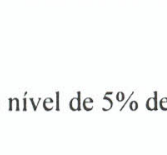 & e. \\
\hline
\end{tabular}




\section{CONCLUSÕES}

Os herbicidas bentazon 400/acifluorfen $170(570,684 \mathrm{e}$ $855 \mathrm{~g} / \mathrm{ha}$ ) e bentazon 400/acifluorfen 140 (540, 648 e $810 \mathrm{~g} / \mathrm{ha})$ isolados e em mistura com óleo mineral (Assist), a $378 \mathrm{~g} / \mathrm{ha}$ foram eficazes no controle do caruru (Amaranthus viridis), joá-de-capote (Nicandra physaloides) e vassoura (Sida rhombifolia) e reúnem condições de praticabilidade para ser utilizados na cultura de soja, em pós-emergência inicial das plantas daninhas latifoliadas mencionadas.

Os herbicidas bentazon 400/acifluorfen 170 e bentazon 400/acifluorfen 140, isolados e em mistura com óleo mineral (Assist), nas doses testadas são seletivos para a cultura da soja, cv. 'Garimpo', em pós-emergência inicial em área total.

\section{LITERATURA CITADA}

BARROS, A. C. de. Eficiência e seletividade de flumicloracpentil e as combinações com outros herbicidas latifolicidas na cultura da soja. In: CONGRESSO BRASILEIRO DE HERBICIDAS E PLANTAS DANINHAS, 19., 1993, Londrina. Resumos dos trabalhos técnico-científicos... Londrina: SBHED, 1993, p. 93-94.

BLANCO, H. G.; Oliveira, D. A.; Araújo, J. B. N.; Grassi, N. Observação sobre o período em que as plantas daninhas competem com a soja (Glycine $\max$ (L.) Merrill. $\mathbf{O}$ Biológico, v. 39, n. 2, 1973. p. 31-35.

BRAGA, O. B. Avaliação de imazethapyr, aplicado em pósemergência, para o controle de plantas daninhas, na cultura da soja (Glycine max). In: CONGRESSO BRASILEIRO DE HERBICIDAS E PLANTAS DANINHAS, 17., 1988, Piracicaba. Resumos... Ribeirão Preto: Ed. Legis Summa Ltda., 1988, p. 152-154.
LACA-BUENDIA, J. P.; REZENDE, A. M. de. Avaliação de herbicidas aplicados em pós-emergência na cultura da soja em Cerrado. In: CONGRESSO BRASILEIRO DE HERBICIDAS E PLANTAS DANINHAS, 19., 1993, Londrina. Resumos dos trabalhos técnico-científicos... Londrina: SBHED, 1993, p. 109.

MACHADO NETO, J.; ANDRADE, T. L. C.; PALMA, V. Efeitos de combinações e misturas de herbicidas de pósemergência na cultura da soja (Glycine $\max$ (L.) Merrill) no controle de plantas daninhas. In: SEMINÁRIO NACIONAL DE PESQUISA DE SOJA. 3.,Campinas, 1984. Resumos... Londrina: EMBRAPA-CNPSo, 1984, p. 81.

MAIA, A.C.; LACA-BUENDIA, J. P.; REZENDE, A. M. de. Avaliação de misturas de herbicidas na cultura de soja (Glycine max (L.) Merrill) cv. UFV-1 em condições de Cerrado. In: SEMINÁRIO NACIONAL DE PESQUISA DE SOJA, 2., 1991, Brasília. Anais... Londrina: EMBRAPA-CNPSo, 1992a, p. 290-298. (EMBRAPACNPSo. Documentos, 1).

MAIA, A. C.; REZENDE, A. M. de; LACA-BUENDIA, J. P. del C. Período crítico de competição de uma comunidade natural de plantas daninhas com a cultura da soja (Glycine max (L.) Merrill), no Triângulo Mineiro. In: SEMINÁRIO NACIONAL DE PESQUISA DE SOJA, 2 ., 1991 Brasília. Anais... Londrina: EMBRAPA-CNPSo, 1992b, p. 370-380. (EMBRAPA-CNPSo. Documentos, 1).

MELHORANÇA, A. L. Efeitos de herbicidas pós-emergentes no desenvolvimento e na produção de grãos de soja (Glycine $\max$ (L.) Merrill). In: SEMINÁRIO NACIONAL DE PESQUISA DE SOJA, 3., Campinas, 1984. Resumos... Londrina: EMBRAPA-CNPSo, 1984, p. 81.

STROHER, I. H.; HADEN, E. Avaliação da eficiência e seletividade de bentazon em mistura com outros herbicidas latifolicidas. In: CONGRESSO BRASILEIRO DE HERBICIDAS E PLANTAS DANINHAS, 19., 1993. Londrina. Resumos dos trabalhos técnicos-científicos... Londrina: SBHED, 1993, p.94-96. 